\title{
Cortisol/DHEA Ratios in Depression
}

We read with interest the study by Young and colleagues (2001). The authors report the results of a study in which plasma cortisol and ACTH were sampled at 10-minute intervals over 24 hours in 25 premenopausal depressed women and a control group matched for age and day of menstrual cycle. No difference in mean ACTH secretion was found between groups and only a trend toward increased cortisol was reported in the depressed group. Pulsatility analyses revealed no significant difference in the number of secretory episodes or half-life of hormone secretion for either ACTH or cortisol, although both average and AUC baseline ACTH was increased. Circadian rhythm of both hormones differed across time, but was not significantly different between groups.

The authors conclude that pulsatile and circadian components of the hypothalamic-pituitary-adrenal (HPA) axis are normal in this patient group and that only $24 \%(6 / 25)$ of depressed women demonstrate hypercortisolemia (defined as the mean plus one standard deviation of the control group). However, the authors have not reported levels of the adrenal androgen dehydroepiandrosterone (DHEA), which recent studies have highlighted as being of relevance, the ratio of cortisol to DHEA being of particular importance.

DHEA is an ACTH-regulated steroid that possesses anti-glucocorticoid properties (Kalimi et al. 1994). The antagonist action of DHEA to cortisol in the brain suggests that measurement of cortisol alone may provide an incomplete estimate of hypercortisolemia (Goodyer et al. 1998). A more precise method of assessing the degree of 'functional' hypercortisolemia is by calculation of the cortisol/DHEA ratio.

A high salivary cortisol/DHEA ratio at presentation in conjunction with disappointing life events has been shown to predict the persistence of major depression (Goodyer et al. 1998). Furthermore, Michael et al. (2000) present data in which the salivary cortisol/DHEA ratio clearly differentiates depressed patients from controls, $82.5 \%$ of depressed subjects having ratios above the $85^{\text {th }}$ percentile of the control group. Therefore decreased levels of DHEA and a consequent elevation of the cortisol/DHEA ratio appears to reflect an additional state abnormality in adult depression.

We propose that future studies of this nature should examine the ratio of cortisol to DHEA, as a more accurate assessment of 'functional' hypercortisolemia.

Peter Gallagher, B.Sc. (Hons) Allan Young, M.B., Ch.B., M.Phil., Ph.D., M.R.C.Psych. University of Newcastle School of Neurosciences and Psychiatry, Newcastle upon Tyne, UK

\section{REFERENCES}

Goodyer IM, Herbert J, Altham PM (1998): Adrenal steroid secretion and major depression in 8- to 16-year-olds. III. Influence of cortisol/DHEA ratio at presentation on subsequent rates of disappointing life events and persistent major depression. Psychol Med 28:265-273

Kalimi M, Shafagoj Y, Loria R, Padgett D, Regelson W (1994): Anti-glucocorticoid effects of dehydroepiandrosterone (DHEA). Mol Cell Biochem 131:99-104

Michael A, Jenaway A, Paykel ES, Herbert J (2000): Altered salivary dehydroepiandrosterone levels in major depression in adults. Biol Psychiatry 48:989-995

Young EA, Carlson NE, Brown MB (2001): Twenty-fourhour ACTH and cortisol pulsatility in depressed women. Neuropsychopharmacology 25:267-276 